\title{
On the linear heat transfer correlation of a heated circular cylinder in laminar crossflow using a new representative temperature concept
}

\author{
An-Bang Wang *, Zdeněk Trávníček ${ }^{1}$ \\ Institute of Applied Mechanics, College of Engineering, National Taiwan University, \\ No. 1, Roosevelt Rd., Sec. IV, 106 Taipei, Taiwan, ROC \\ Received 3 October 2000; received in revised form 8 March 2001
}

\begin{abstract}
Determination of the reference temperature for the kinematic viscosity of Reynolds number is the key problem for describing the forced convection of a heated bluff-body. The heat transfer of a heated circular cylinder in laminar cross airflow is experimentally investigated in this paper. New correlation equations based on the effective temperature have been firstly derived. All experimental data collapsed excellently on the correlation curves. Moreover, the derived Nusselt and Reynolds number relations are independent of the vortex shedding patterns. The correlation equations have further been transformed to an explicit form without temperature ratio. By introducing a newly proposed representative Reynolds number concept, linear correlation equations have been successfully derived. (c) 2001 Elsevier Science Ltd. All rights reserved.

Keywords: Heated circular cylinder; Laminar flow; Heat transfer correlation; Vortex shedding patterns; Effective temperature; Representative temperature
\end{abstract}

\section{Introduction}

\subsection{Reference temperature and parallel vortex shedding of a heated cylinder}

The forced convection of a heated circular cylinder in laminar crossflow has long been studied. The Reynolds number, $R e$, plays an important role for the description of flow and heat transfer character, and is commonly defined as $R e=U_{\infty} d / v$, where $U_{\infty}$ is the free-stream velocity, $d$ is the cylinder diameter and $v$ is the kinematic viscosity. Generally speaking, the free-stream velocity is independent of the cylinder temperature. The cylinder

\footnotetext{
${ }^{*}$ Corresponding author. Tel.: +886-2-2363-0979 ext. 405; fax: $+886-2-2367-8001$.

E-mail address: abwang@spring.iam.ntu.edu.tw (A.-B. Wang).

${ }^{1}$ Present address: Institute of Thermomechanics, Academy of Sciences of the Czech Republic, Prague, Dolejškova 5, 18200 Prague 8, Czech Republic.
}

diameter could be also considered as a constant for the relatively small thermal expansion in comparison with the original diameter in most heating experiments. The temperature variation between the fluid and cylinder wall leads to appreciable changes of the kinematic viscosity of fluid, so that the local Reynolds number varies significantly in the flow field, especially in the near field of the heated cylinder. Determination of a reference (or characteristic) temperature for the kinematic viscosity $v$ to calculate the reference (or characteristic) Reynolds number for describing the flow is the first problem of studying the flow around a heated cylinder.

Different reference temperatures $T_{\text {ref }}$ have been proposed to account for the effects of above-mentioned temperature change of the heated cylinder in the literature. The basic equation for the reference temperature could be written as a linear dimensionless equation of the cylinder wall temperature $T_{\mathrm{W}}$ and the free-stream temperature $T_{\infty}$ and represented as

$T_{\mathrm{ref}} / T_{\infty}=1+c\left(T_{\mathrm{W}}-T_{\infty}\right) / T_{\infty}$ 


\begin{tabular}{|c|c|c|c|}
\hline \multicolumn{2}{|c|}{ Nomenclature } & $z$ & $\begin{array}{l}\text { coordinate, along the cylinder axis (m) } \\
\text { (see Fig. 1) }\end{array}$ \\
\hline$d$ & cylinder diameter $(\mathrm{m})$ & \multicolumn{2}{|c|}{ Greek symbols } \\
\hline$f$ & vortex shedding frequency & $\beta$ & coefficient of the thermal expansion of \\
\hline$g$ & acceleration of gravity & & the air $\left(=0.00333 \mathrm{~K}^{-1}\right)$ \\
\hline$G r$ & Grashof number, $g \beta\left(T_{\mathrm{W}}-T_{\infty}\right) d^{3} / v^{2}$ & $\varepsilon$ & emissivity of a cylinder surface \\
\hline$h$ & $\begin{array}{l}\text { mean heat transfer coefficient }\left(\mathrm{W} \mathrm{m}^{-2} \mathrm{~K}^{-1}\right) \\
\text { electrical current }\end{array}$ & $v$ & kinematic viscosity at the upstream \\
\hline$k$ & thermal conductivity $\left(\mathrm{W} \mathrm{m}^{-1} \mathrm{~K}^{-1}\right.$ ) & & temperature $\left(\mathrm{m}^{2} \mathrm{~s}^{-1}\right)$ \\
\hline$l$ & length of the test region of cylinder $(\mathrm{m})$ & $\sigma$ & $\begin{array}{l}\text { Stefan-Boltzman constant } \\
\left(=5.6705 \times 10^{-8} \mathrm{~W} \mathrm{~m}^{-2} \mathrm{~K}^{-4}\right)\end{array}$ \\
\hline & $\begin{array}{l}\text { mean Nusselt number, } h d / k \\
\text { oblique vortex shedding }\end{array}$ & \multicolumn{2}{|c|}{ Subscripts } \\
\hline$P$ & heat transfer rate & $\mathrm{C}$ & critical stage - the boundary between the \\
\hline $\begin{array}{l}\text { Pr } \\
\text { PVS }\end{array}$ & $\begin{array}{l}\text { Prandtl number } \\
\text { parallel vortex shedding }\end{array}$ & & $\begin{array}{l}\text { laminar steady recirculation regime and the } \\
\text { laminar vortex shedding regime }\end{array}$ \\
\hline$R e$ & Reynolds number, $U_{\infty} d / v$ & $\mathrm{CC}$ & control cylinders \\
\hline St & Strouhal number, $f d / U_{\infty}$ & $\mathrm{CON}$ & conduction \\
\hline$T$ & temperature $(\mathrm{K})$ & CONV & convection \\
\hline$T^{*}$ & temperature ratio, $T_{\mathrm{W}} / T_{\infty}(\mathrm{K} / \mathrm{K})$ & eff & effective \\
\hline$U$ & fluid velocity $\left(\mathrm{m} \mathrm{s}^{-1}\right)$ & $\mathrm{f}$ & film \\
\hline$V$ & voltage across cylinder test part & RAD & radiation \\
\hline$x$ & $\begin{array}{l}\text { coordinate, toward the downstream direc- } \\
\text { tion (m) (see Fig. 1) }\end{array}$ & $\begin{array}{l}\text { ref } \\
\text { rep }\end{array}$ & $\begin{array}{l}\text { reference } \\
\text { representative properties }\end{array}$ \\
\hline$y$ & coordinate, perpendicular to the cylinder & $\mathrm{W}$ & cylinder wall \\
\hline & axis $(\mathrm{m})$ (see Fig. 1) & $\infty$ & free-stream \\
\hline
\end{tabular}

where $c$ is the proportional coefficient and all temperatures in Eq. (1) are in K. Different $c$-values ranging from 0 to 1.0 have been chosen by different authors. Typically, the fluid properties have been calculated directly from the free-stream temperature $(c=0)$ by Zukauskas and Ziugdza [1] or based on the cylinder wall temperature $(c=1)$ by Lange et al. [2], however, the derived results are temperature-dependent even for small temperature ratios $\left(T^{*} \equiv T_{\mathrm{W}} / T_{\infty} \leqslant 1.5\right)$. It is worthy to note that the term $T^{*}$ is sometimes referred to as "temperature loading" (or "temperature loading factor") in the literature. This terminology is not used here to avoid misunderstanding the term as the thermally induced stress. The socalled "film" temperature $T_{\mathrm{f}}$, defined as the arithmetic mean of the cylinder wall temperature $T_{\mathrm{W}}$ and the freestream temperature $T_{\infty}$ and corresponding to the $c$-value of 0.5 in Eq. (1), is the most frequently used artificial temperature in the literature to calculate the kinematic viscosity [3-5]. However, no physics of fluid mechanics has been introduced in the derivation process of the film temperature. Other possibilities to get the value of kinematic viscosity are to use more than one value of $c$, e.g. the free-stream temperature for the density, but the film temperature for other fluid properties [6,7].

Recently, a so-called "effective temperature" has been introduced to study the shedding frequency from a heated circular cylinder by Wang et al. [8]. The effective temperature equation is derived from a similarity concept that the effective critical Reynolds number $R e_{\mathrm{C}, \text { eff }}$, which employs a kinematic viscosity computed from the effective temperature equation, for the onset of vortex shedding from a cylinder is the same for both heated and unheated cases [8-10]. Based on this assumption, the constant $c$ of Eq. (1) was determined from experiments for the temperature range of $T^{*}<2.0$ and the effective temperature $T_{\text {eff }}$ was derived as

$T_{\text {eff }} / T_{\infty}=1+0.28\left(T_{\mathrm{W}}-T_{\infty}\right) / T_{\infty}$

by Wang et al. [8]. It is worthy to note that the validity range of Eq. (2) could be extended for still higher temperature range as discussed in [8].

It has been well documented in $[11,12]$ that the vortex shedding from a circular cylinder becomes not parallel but oblique to the cylinder axis under the influence of the so-called end effect caused by the end plates of the test cylinder. Different end-manipulating methods to isolate the end-effects from the tested region of the cylinder have been developed for the iso-thermal flows [1315]. However, not much attention has been drawn to the cases of heated cylinder until recently. Wang et al. [8] have successfully developed the construction (see also Fig. 1(a)) and generated the approved two-dimensional parallel vortex shedding (PVS) from a heated cylinder for $T^{*}<2.0$. 
(a) Model A

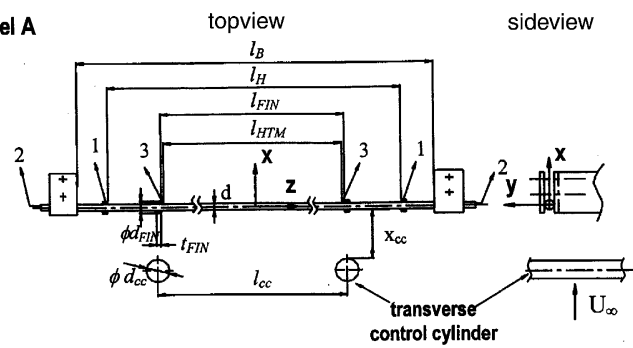

(b) Model B

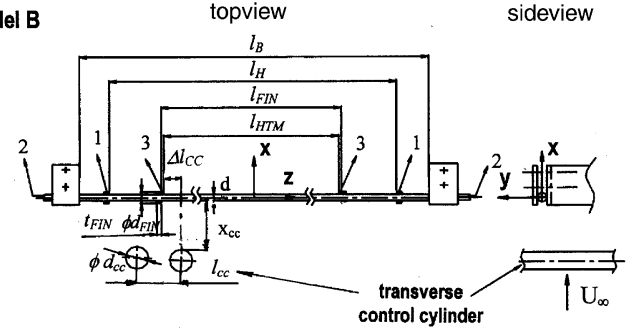

Fig. 1. Scheme of the test models, (a) model A for generation of parallel vortex shedding and (b) model B for generation of oblique vortex shedding. Here $d=1.07 \mathrm{~mm}, d_{\mathrm{CC}}=6.0 \mathrm{~mm}, l_{\mathrm{HTM}}=128 \mathrm{~mm}, l_{\mathrm{FIN}}=130.6 \mathrm{~mm}, t_{\mathrm{FIN}}=2.0 \mathrm{~mm}, l_{\mathrm{H}}=326 \mathrm{~mm}, l_{\mathrm{B}}=352 \mathrm{~mm}$, $x_{\mathrm{CC}}=11-12 \mathrm{~mm}$. For model A, $l_{\mathrm{CC}}=132 \mathrm{~mm}$. For model $\mathrm{B}, l_{\mathrm{CC}}=19 \mathrm{~mm}$ and $\Delta l_{\mathrm{CC}}=6 \mathrm{~mm}$. 1: connection to power supply, 2: to a thermocouple, 3: to a voltmeter.

The relationship of Strouhal number (defined as $S t=f d / U_{\infty}$, where $f$ is the vortex shedding frequency) and Reynolds number is used to quantitatively measure the parallel vortex shedding frequency from a cylinder. For non-isothermal cases, Wang et al. [8] and Yahagi [16] have shown that the frequency of vortex shedding (or $S t$-value) decreases with the increasing cylinder temperature. However, the former paper revealed that all experimental PVS data of the heated and unheated circular cylinders collapsed to a "universal" $S t-R e_{\text {eff }}$-curve for different temperature ratios by using the $R e_{\text {eff }}$-concept. Here, $R e_{\text {eff }}$ was denoted as $R e_{\text {eff }}=U_{\infty} d / v_{\text {eff }}$, and the $v_{\text {eff }}$-value was calculated based on the effective temperature of Eq. (2). The $S t-R e_{\text {eff }}$-curve was written in [8] as:

$S t=0.2660-\frac{1.0160}{\sqrt{R e}_{\text {eff }}}$.

The maximum deviation of Eq. (3) from the Williamson and Brown's $S t-R e$-formula [17] was less than $0.23 \%$ in the laminar vortex shedding regime for $R e \leqslant 180$ (see Wang et al. [8]). However, to the best of the authors' knowledge, the performance of the effective temperature concept has not been tested for describing the heat transfer correlation of a heated cylinder in a laminar crossflow yet, except the work of Dumouchel et al. [10]. However, the $T^{*}$-range is narrower (less than 1.5) and the proportional coefficient for the effective temperature equation is a bit smaller $(c=0.24)$ in [10]. This is thus the first motivation of the present work.

\subsection{Forced convection correlations of a heated cylinder in crossflow}

To avoid the influence (or "contamination") of free convection, it is necessary to determine where the lower limit of the forced convection regime is. There have been different criteria in the literature to determine the forced convection region based on the ratio of $G r / R e^{s}$, where $G r=g \beta\left(T_{\mathrm{W}}-T_{\infty}\right) d^{3} / v_{\infty}^{2}$ is the Grashof number, $g$ is the acceleration of gravity, and $\beta$ is the coefficient of the thermal expansion. Typically used value of exponent $s$ was within the range of 1.8-3. As a commonly used example, a criterion for the forced convection is in the form of $G r / R e^{1.8}<0.58 \pm 0.13$ by Sharma and Sukhatme [18]. Another frequently adapted form is $G r / R e^{2}$, which is sometimes called the Richardson number $R i,<0.5$. This was used as the criterion for the forced convection in [3]. Another form with $G r / R e^{2.39}<0.21$ was proposed in [4] after simple modification for the air. Further different criterion of $G r / R e^{3}<1$ was proposed in [5] for the micro-sized heated cylinder (i.e. hot wire, with cylinder diameter from 0.003 to $0.054 \mathrm{~mm}$ ).

One of the most commonly used relations in the literature for the forced convection of a heated circular cylinder in crossflow is the theoretically proposed King's law in 1914 - see, e.g. [19]. It is simply a square-root dependence of the Nusselt number on the Reynolds number. The Nusselt number $N u$ is defined as $N u=h d / k$, where $h$ is the mean heat transfer coefficient over the whole cylinder surface and $k$ is the thermal conductivity of the fluid. With a slightly modified form, the mean heat transfer of a heated cylinder has also been expressed as the relations between the Nusselt number and an additional parameter, Prandtl number, as

$N u=\left(A \operatorname{Pr}^{p}+B \operatorname{Pr}^{q} \operatorname{Re}^{n}\right)$,

where $A, B, n, p, q$ are experimentally determined constants and $P r$ is the Prandtl number. According to Hilsenrath and Touloukian [20], the $\mathrm{Pr}$-value monotonically decreases from 0.71 at $20^{\circ} \mathrm{C}$ to 0.68 at $300^{\circ} \mathrm{C}$, for airflow at atmospheric pressure, the $\mathrm{Pr}$-values are sometimes approximated as a constant of 0.7 .

Some other efforts have been made to implicitly express the effect of temperature ratio $T^{*}$ in the heat transfer relation by using different reference temperatures. However, none of those efforts are able to really account for the influence of temperature ratios except for moderate $T^{*}$-range where the effect of temperature ratio is insignificant. Therefore, the commonly used heat transfer relationship are written in the explicit 
form of temperature ratio for larger $T^{*}$-values (say $T^{*}>1.5$ ). In these cases, the fluid properties calculated for the Nusselt and Reynolds numbers are commonly based on the film temperature and the equations were in the form

$N u_{\mathrm{f}}=\left(A+B R e_{\mathrm{f}}^{n}\right) F^{r}$,

where $N u_{\mathrm{f}}=h d / k_{\mathrm{f}}$ and $R e_{\mathrm{f}}=U_{\infty} d / v_{\mathrm{f}}$. The factor $F$ is based either on the ratio of $T_{\mathrm{f}} / T_{\infty}$ (e.g. [4,5,21], and recently [2]) or $T^{*}$, or the ratio of $T_{\mathrm{W}} / T_{\infty}$ (e.g. [3,22]). The empirical constant $r$ has mostly taken as 0.17 for the former case (except $q=0.154$ was used in [4]), and $r=n / 4$ for the latter cases. A large number of investigators have evaluated the constants of Eq. (5). The representative collections could be found in the excellent review of Morgan [3] or other publications - e.g. $[1,2,23]$. The values of empirical constants $A, B$ and $n$ were suggested by Hilpert [22] and Morgan [3] as $A=0$, $B=0.795, n=0.384$ for the laminar steady recirculation regime, and $A=0, B=0.583, n=0.471$ for laminar vortex shedding regime, respectively. The border of both regimes was at $R e_{\mathrm{f}}=35$.

However, as shown in [8], the critical Reynolds number (i.e. the border of both regimes) is a function of temperature ratio, except the calculation of the Reynolds number is based on the effective temperature. This is also one reason to encourage us by using the effective Reynolds number for describing the heat transfer correlation equation from this aspect. Moreover, since in most tests the wake from the heated cylinder might not be truly two-dimensional, it is important to examine the influence of the three-dimensional vortex shedding pattern on the heat transfer of the heated cylinder. Therefore, another motivation of this paper is to investigate the effect of three-dimensional wake pattern on the heat transfer correlation of a heated cylinder.

\subsection{Plan of the paper}

In Section 3.1, the flow and heat transfer characters of the two-dimensional wake (or PVS) from a heated cylinder are investigated for temperature ratio $T^{*}=1.1-1.8$. Attention is focused on the availability of the effective temperature concept for the Nusselt and Reynolds numbers relationship. The generation of oblique vortex shedding (OVS) from the heated cylinder is discussed in Section 3.2 for the flow and heat transfer characters. The performance of the effective temperature concept is tested for the heat transfer of cylinder in the OVS mode. In Section 3.3, a further attempt is made to eliminate the temperature ratio from the heat transfer correlation equation. A linear form of heat transfer correlation for a heated cylinder is derived based on a newly proposed representative temperature concept.

\section{Experimental descriptions}

\subsection{Facilities and measuring techniques}

Experiments were made in a very low speed wind tunnel, which has a rectangular test section $(0.5 \mathrm{~m}$-high, $0.7 \mathrm{~m}$-width and $0.9 \mathrm{~m}$-long). The velocity range was from 0.07 to $2.4 \mathrm{~m} / \mathrm{s}$ with maximal non-uniformity less than $2 \%$. The turbulence intensity was approximately $0.2 \%$ for most experiments, and less than $0.7 \%$ for experiments at velocity less than $0.2 \mathrm{~m} / \mathrm{s}$. The investigated $R e$-range from 5 to 163 covered the whole laminar flow regime, i.e. laminar steady recirculation regime as well as laminar vortex shedding regime where the Kármán vortex street was observed.

The smoke-wire technique was used for the visualization of wake flow behind the test cylinder. The smoke-wire was uniformly twisted from three resistance wires with $0.1 \mathrm{~mm}$ in diameter, and was connected to a direct current supply. A stroboscope light was used for the illumination of flow visualizations. Pictures were taken with single shot of flashlight from the top view. The camera was Nikon F-801s equipped with the lens Nikon AF Zoom-Nicker 35-70 mm.

A one-component TSI laser doppler anemometer (LDA) was used to measure the free-stream velocity in forward-scattering mode by a $60 \mathrm{~mW}$ Helium-Neon Laser. No artificial seeding has been added in experiments (see [24]). The optical measuring volume was $\phi 0.169 \mathrm{~mm} \times 1.97 \mathrm{~mm}$ and located at $x=-56 d, y=0$, $z=0$ upstream of the cylinder. The blockage ratio was $5 \%$ and the measured velocity was corrected by using the correction factor $c_{\mathrm{B}}=1.023$ according to Morgan [3].

The cylinder was made from a smooth stainless steel tube, its outside and inside diameters were 1.07 and 0.77 $\mathrm{mm}$, respectively. The cylinder was mounted horizontally in the middle of the test section. Two test models, as shown in Figs. 1(a) and (b), were used in the present study. Model A was designed to produce two-dimensional wake (or parallel vortex shedding) behind the cylinder. Model B was designed to generate the threedimensional wake (or OVS) by using artificially asymmetric boundary conditions. (It is worthy to note here that the wake behind model B still remained PVS for $R e_{\text {eff }}<63$ and became OVS for higher $R e_{\text {eff }}$-range.)

The cylinder was heated by Joule effect of direct current from the contact points denoted as position 1 as shown in Fig. 1. The heating voltage across the cylinder test part, which was necessary for evaluating the heat transfer rate, was measured from the contact points denoted as position 3 . Each connection was made from a stainless steel wire of diameter $0.2 \mathrm{~mm}$, welded on the rear side of cylinder surface and "hid" itself in the wake from the upstream control cylinder. No influence of connected wires on the parallel vortex shedding of cylinder wake in the test region has been found. One pair of 
cooling brass fins was mounted in the wakes from the upstream transverse control cylinders to stabilize the two-dimensional wake of heated cylinder. The fins were able to slide on the cylinder, and the interface between them was filled with the silicon heat sink compound that gave good heat conduction and was helpful for damping the vibration of fins. The value of $l_{\mathrm{CC}} / d$ was 123 for model $\mathrm{A}$ and about 18 for model $\mathrm{B}$. The coordinate system is also shown in Fig. 1. The origin of the coordinates was in the middle of the test-cylinder and the flow was from the bottom to the top in the figure.

The cylinder temperature was measured by an ironconstantan thermocouple that was plugged in the tested circular cylinder tube. The bare wire of the thermocouple had diameter of $0.12 \mathrm{~mm}$ and was insulated by Teflon-layer with total diameter around $0.27 \mathrm{~mm}$. The maximum temperature ratio was around 2 (the maximum $T_{\mathrm{W}}$ was about $350^{\circ} \mathrm{C}$ ) to derive the critical Reynolds number for the onset of vortex shedding. However most experiments were made until $T^{*}=1.80$ for the heat transfer measurements. Similar to Hatton et al. [4] and Dumouchel et al. [10], the temperature difference between inside and outside of cylinder surface has been neglected since the cylinder wall had only $0.15-\mathrm{mm}$ thickness. Therefore, the measured temperature by the thermocouple was considered as the average surface temperature of the cylinder, $T_{\mathrm{W}}$. The thermometers have been calibrated and the maximum measuring uncertainties of the $T_{\mathrm{W}}$ and $T_{\infty}$ were around \pm 1.7 and $\pm 0.8 \mathrm{~K}$, respectively. Two metal brackets were used to fix the cylinder at both ends of supporting frame. Adequate tension stress has been preloaded on the cylinder by these two brackets in cold stage in order to avoid bowing of the cylinder axis after heating. Glass fabric layers (about $1 \mathrm{~mm}$ thick) were used as the insulator between the cylinder and the brackets. According to the results of Wang et al. [8], the generation of the PVS wake was independent on the cylinder heating, and the maximum temperature difference across the cylinder span was evaluated around $12 \%$ for the temperature ratio of $T^{*}=1.9$.

To quantitatively test the influence of vortex shedding mode on the flow and heat transfer characters of the heated cylinder, Model B was developed to generate OVS from cylinder by using non-symmetrical arrangement of transverse control cylinders as shown in Fig. 1(b). Models B and A were basically identical except the locations of the transverse control cylinders. It is worthy to note here that the pattern of OVS could also be achieved by simply removing one of the control cylinders out of tunnel test-section. But model B was finally adopted in the experiments under the following considerations: (a) the identical blockage ratio and (b) similar heat-loss conditions of the heated cylinder in the wakes of two control cylinders for models A and B. The former is straightforward. The latter was achieved by placing one control cylinder inside of the tested region (within the region of $z=-0.5 l_{\text {HTM }}$ in Fig. 1(b)) and one control cylinder outside of it. The total wake regions from the upstream control cylinders on the heated cylinder for model B were arranged approximately the same as those for model A.

\subsection{Heat transfer evaluations}

The electric heating rate of a cylinder within the heated region (the length of $l_{\mathrm{H}}$ in Fig. 1) was calculated as the product of current $I$ and voltage $V$. The thermal equilibrium equation per unit length is expressed as the sum of the heat convection, heat radiation and heat conduction

$\frac{I V}{l}=\frac{P_{\mathrm{CONV}}}{l}+\frac{P_{\mathrm{RAD}}}{l}+\frac{P_{\mathrm{COND}}}{l}$,

where $P_{\mathrm{CONV}}, P_{\mathrm{RAD}}$ and $P_{\mathrm{COND}}$ are heat loss of heat convection, heat radiation and heat conduction, respectively. The heat convection is obtained as $h \pi d l\left(T_{\mathrm{W}}-T_{\infty}\right)$ for the forced convection discussed in this paper, where $h$ is the heat transfer coefficient. The heat transfer coefficient $h$ can be further expressed by in terms of Nusselt number $\left(h=N u_{\mathrm{f}} k_{\mathrm{f}} / d\right)$. The heat radiation loss is generally described as $P_{\mathrm{RAD}}=$ $\pi d l \varepsilon \sigma\left(T_{\mathrm{W}}^{4}-T_{\infty}^{4}\right)$, where $\varepsilon$ and $\sigma$ are the cylinder surface emissivity and Stefan-Boltzman constant, respectively. The $P_{\text {COND }}$ could be estimated by using one-dimensional heat conduction analysis from the temperature gradient at both ends of heated cylinder. The resultant $P_{\mathrm{COND}^{-}}$ value for model A was estimated to be less than $1.5 \%$ of the convective heat transfer in [8] and was thus neglected in this paper. Hence, Eq. (6) could be written in the following form:

$N u_{\mathrm{f}}=\frac{1}{\pi k_{\mathrm{f}}\left(T_{\mathrm{W}}-T_{\infty}\right)}\left[\frac{I V}{l}-\pi d \varepsilon \sigma\left(T_{\mathrm{W}}^{4}-T_{\infty}^{4}\right)\right]$.

To simulate the two-dimensional heat transfer, special care has been taken to keep the temperature distribution uniform along the cylinder span. Fig. 2 shows the typically measured temperature distributions of test cylinder in the steady recirculation regime $(R e=25)$ and in the periodic wake regime $(R e=149)$ for $T^{*}=1.5$. For higher Reynolds number, the temperatures were almost constant along the whole cylinder test region beyond a distance shorter than $5 \%$ of $l_{\mathrm{CC}}$ from both ends near the fins. The lower temperature region became a bit wider for $R e=25$. The lowest $T^{*}$-values along the cylinder were about 0.95 for both cases and occurred at the positions of the fins, which were located at $x= \pm 0.5 l_{\mathrm{CC}}$. The maximum temperature deviation from the $T^{*}(z=0)$ was within $3.5 \%$ at both ends of the cylinder test region for $T^{*}=1.5$ as shown in Fig. 2. The maximum temperature difference was around 7\% (not shown here) for the highest temperature ratio in the present study. Since 


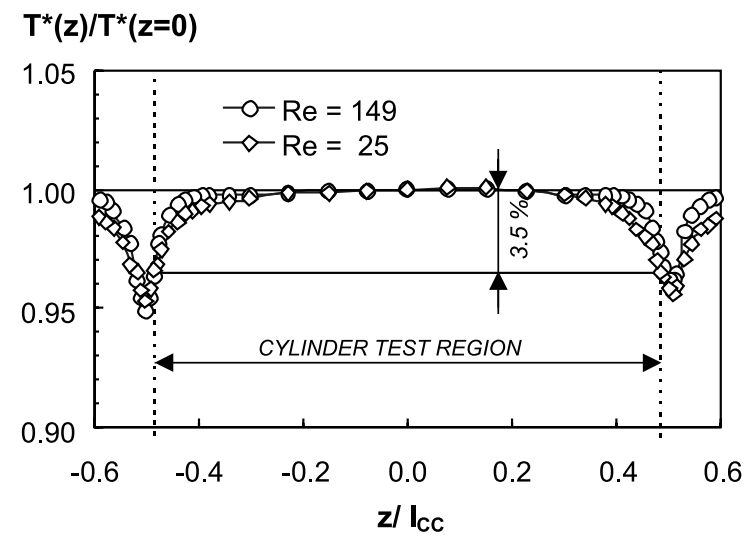

Fig. 2. Temperature distribution along the cylinder axis for model A at the $T^{*}=1.5$.

the temperature non-uniformity for evaluation of the overall heat transfer of the cylinder is small, the influence of temperature non-uniformity can thus be considered as negligible.

In this paper, the value of $k_{\mathrm{f}}$ was calculated from the film temperature $T_{\mathrm{f}}$ in $\mathrm{K}$ by using the formula given by Hilsenrath and Touloukian [20]. The formula was converted to the SI-units and written as

$k_{\mathrm{f}}=\frac{0.002648 \sqrt{T_{\mathrm{f}}}}{1+\left(\left(254.4 \times 10^{-12 / T_{\mathrm{f}}}\right) / T_{\mathrm{f}}\right)}$.

In comparison with some other $k_{\mathrm{f}}$-values used in the literature, Eq. (8) gives $k_{\mathrm{f}}$-values $1.0-1.5 \%$ lower than those from [25] for the temperature range of $20-300^{\circ} \mathrm{C}$. The formula used in [5] gives roughly the same $k_{\mathrm{f}}$-values as those from Eq. (8) for temperature around $150^{\circ} \mathrm{C}$, but much lower $k_{\mathrm{f}}$-values for still higher temperatures (about $5 \%$ lower at $300^{\circ} \mathrm{C}$ ). Provided that the uncertainty of thermal conductivity $k_{\mathrm{f}}$ calculated from Eq. (8) is within $\pm 2 \%$, the uncertainty of $N u_{\mathrm{f}}$-values calculated from Eq. (7) could be estimated as less than $\pm 5 \%$ at $95 \%$ confidence level.

The first term in the bracket on the right-hand side of Eq. (7) is the cylinder heating per unit length and ranged from 0 (for unheated case) to $160 \mathrm{~W} / \mathrm{m}$ in the present experiment. The maximum uncertainty of the current and voltage measurement is less than $\pm 0.7 \%$, and $\pm 0.3 \%$, respectively. The second term, the heat-flux loss due to radiation, is usually considered negligible provided that the $T_{\mathrm{W}}$ does not exceed $300^{\circ} \mathrm{C}$ ) (see, e.g. [26]). This approximation is valid for cases of platinum and tungsten wires, but seems to be too rough for the stainless steel tube. The maximal radiation loss is calculated as about $6 \%$ of the heat convection in the present study. This is due to the higher emissivity of stainless steel tube (i.e. $\varepsilon_{\text {steel }}=0.2$ for lightly oxidized surfaces and $\varepsilon_{\text {tungsten }}=0.04$ based on Incropera and DeWitt [27]).
To avoid the influence from mixed convection, all the present data used for the heat transfer correlation satisfy the criteria of $G r / R e^{1.8}<0.62, \quad R i<0.5, \quad$ and $G r / R e^{2.39}<0.21$ (according to Sharma and Sukhatme [18], Morgan [3], and Hatton et al. [4], respectively).

\section{Results and discussions}

\subsection{Two-dimensional wake and heat transfer of a heated cylinder}

Experiments were first conducted using model A to produce parallel vortex shedding from the tested cylinder. Since the kinematic viscosity is a function of temperature, the subscript of the Reynolds number is used for the referred temperature in this paper, e.g. $R e_{\infty}=U_{\infty} d / v_{\infty}$, where $v_{\infty}$ is the kinematic viscosity based on the free-stream temperature $T_{\infty}$. However, for simplicity, the subscript " $\infty$ " will sometimes be omitted in this paper, i.e. $R e=R e_{\infty}$, when there is no confusion. Fig. 3 shows the visualization of flow pattern behind model A for the conditions of $T^{*}=1.9$ and $R e=108$. This picture was taken from the top-view of $x-z$-plane and the flow was from right to the left. It revealed a stable parallel vortex shedding pattern behind a heated cylinder.

In order to have a quantitative comparison with the existing formula in the literature, the present data of heat transfer from two-dimensional wake are first expressed in terms of film Nusselt number $\mathrm{Nu}_{\mathrm{f}}$ in Fig. 4. The $N u_{\mathrm{f}}$-values are calculated from Eq. (7). It shows clearly in this figure that the data of $N u_{\mathrm{f}}-R e$-relationship (solid symbols) spread over much wider region than those of $N u_{\mathrm{f}}-R e_{\mathrm{f}}$-relationship (empty symbols) for three temperature ratios, $T^{*}=1.10,1.50$ and 1.80 , respectively. Moreover, two $N u_{\mathrm{f}}-\operatorname{Re}_{\mathrm{f}}$-curves for $T^{*}=1.10$ and 1.80, calculated from the correlation of Morgan [3], are also plotted in Fig. 4. In general, the agreement of present data with either curve is good. The maximum deviations are from $-4.0 \%$ to $+2.4 \%$ and the S.D. is $1.6 \%$. Further comparisons of present data were made with those from $[2,4,5,21,23,25]$, but are not shown here. The agreement with a correlation from [2] is very good and the differences vary from $-2 \%$ to $+4 \%$. The agreement with $[4,5,20,25]$ is satisfactory and the differences are within $\pm 9 \%$. Larger differences are found between the present data and the correlation in [23] (from $-3 \%$ to $+12 \%)$. The discrepancy suggests the possible influences of other parameters, such as temperature ratio, flow and thermal boundary conditions, etc.

In the literature, the heat transfer formula is commonly expressed as either (a) by one continuous correlation equation for the whole investigated range or (b) by different correlation equations in individual characteristic flow regimes. Generally, the former is convenient 


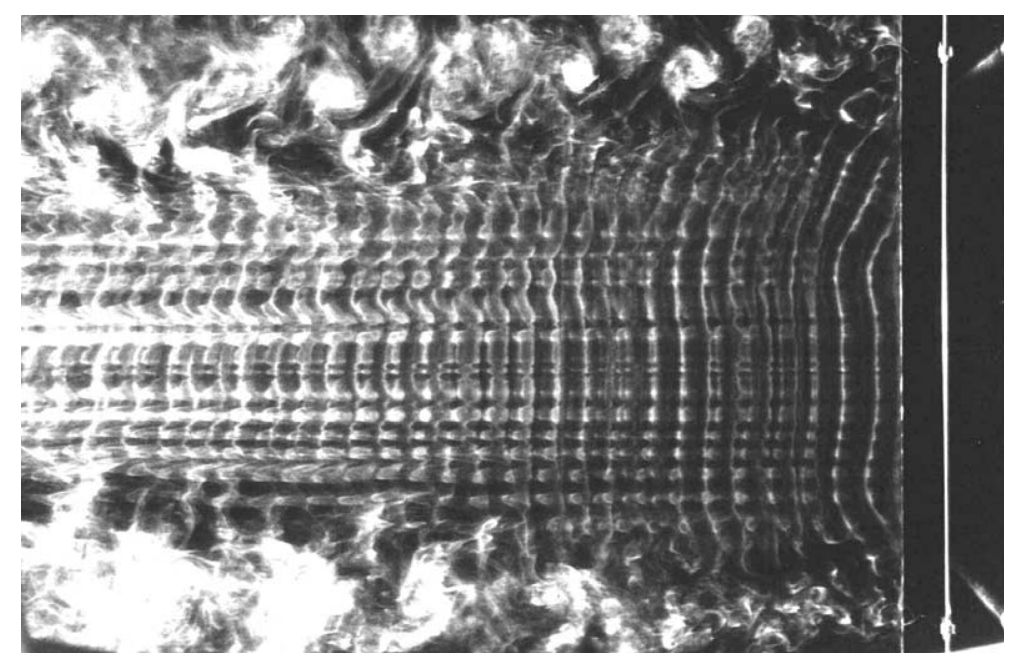

Fig. 3. Smoke-wire visualization of parallel vortex shedding mode from a heated cylinder, $R e_{\text {eff }}=73, T^{*}=1.9$, $\left(\operatorname{Re}=108, T_{\mathrm{W}}=300^{\circ} \mathrm{C}\right)$.

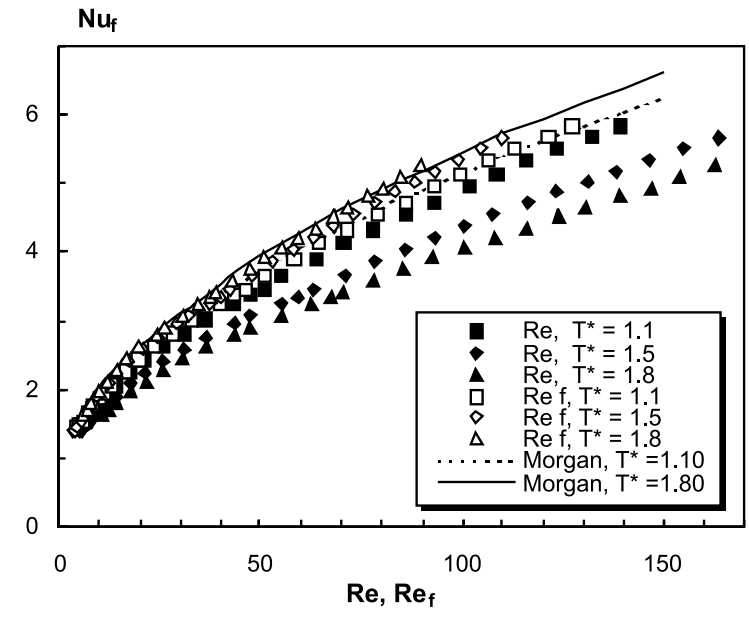

Fig. 4. $N u_{\mathrm{f}}-R e$ - and $N u_{\mathrm{f}}-R e_{\mathrm{f}}$-relationship for heat transfer of a parallel vortex shedding from a heated cylinder. The curves are $N u_{\mathrm{f}}-R e_{\mathrm{f}}$-relationship from [3].

for use and is thus preferred for the engineering applications. The latter normally has higher accuracy and may also represent the individual physical nature in each flow regimes. Based on these considerations, two correlation equations are used for fitting experimental data in two regimes separated by the critical Reynolds number for the onset of vortex shedding in this section. In Section 3.2, for application and comparison purposes, a single continuous correlation equation will be used to describe all PVS and OVS data in the whole laminar range.

The value of critical Reynolds number for the onset of vortex shedding, based on the free-stream tempera- ture and denoted as $R e_{\mathrm{C}}$, has the value of 47.7 for the unheated case. The $R e_{\mathrm{C}}$-values increased with the increasing wall temperature up to 72.2 for $T^{*}=1.96$. The $R e_{\mathrm{C}, \mathrm{f}}$-values were also $T^{*}$-dependent and ranged from 37 (for $T^{*}=1.8$ ) to 46 (for $T^{*}=1.1$ ) if the viscosity was evaluated by the film temperature. However, the effective critical Reynolds number, $R e_{\mathrm{C} \text {,eff }}$, was found to be a constant of $47.5 \pm 0.7$ for $T^{*}<2$ using the effective Reynolds number concept (see [8] in detail). Therefore, the validity border in the following heat transfer correlations is all expressed in terms of $R e_{\mathrm{C}, \text { fff }}$ for simplicity.

Fig. 5(a) shows experimental data of $T^{*}=1.1,1.5$ and 1.8 for heat transfer in the steady recirculation regime. The variable of $R e_{\mathrm{f}} T^{* 0.25}$ in $x$-axis has been directly used to include the influence of heating temperature according to Morgan [3]. All experimental data collapsed excellently together in Fig. 5(a) in comparison with those in Fig. 4. By using the least-squares fitting, the forced convective heat transfer in the steady recirculation flow regime can be described as

$N u_{\mathrm{f}}=0.502+0.434\left(\operatorname{Re}_{\mathrm{f}} T^{* 0.25}\right)^{0.5}$,

for $R e_{\text {eff }}<R e_{\mathrm{C} \text {,eff }}=47.5 \pm 0.7$ (or approximately for $R e_{\mathrm{f}} T^{* 0.25}<45 \pm 2$ ) and $1.1 \leqslant T^{*} \leqslant 1.8$. Eq. (9) is displayed as the solid line in Fig. 5(a). It is important to note here that the power series form of the variable $\left(\operatorname{Re}_{\mathrm{f}} T^{* 0.25}\right)^{0.5}$ was used here as the basic form for the curve fitting of experimental Nusselt number data. A two-term truncated series was used in this section for simplicity. The maximum and S.D. of experimental data from Eq. (9) are $1.9 \%$ and $0.6 \%$, respectively. The first term on the right-hand side of Eq. (9), the constant 0.502 , could be regarded as the "virtual origin" of $N u_{\mathrm{f}}-\operatorname{Re}_{\mathrm{f}} T^{* 0.25}$ curve for $R e_{\mathrm{f}}=0$. No further physical 


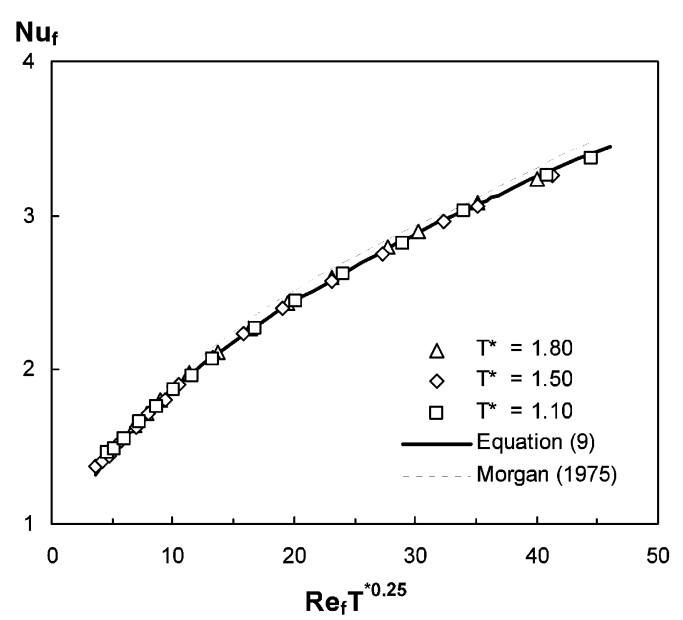

(a)

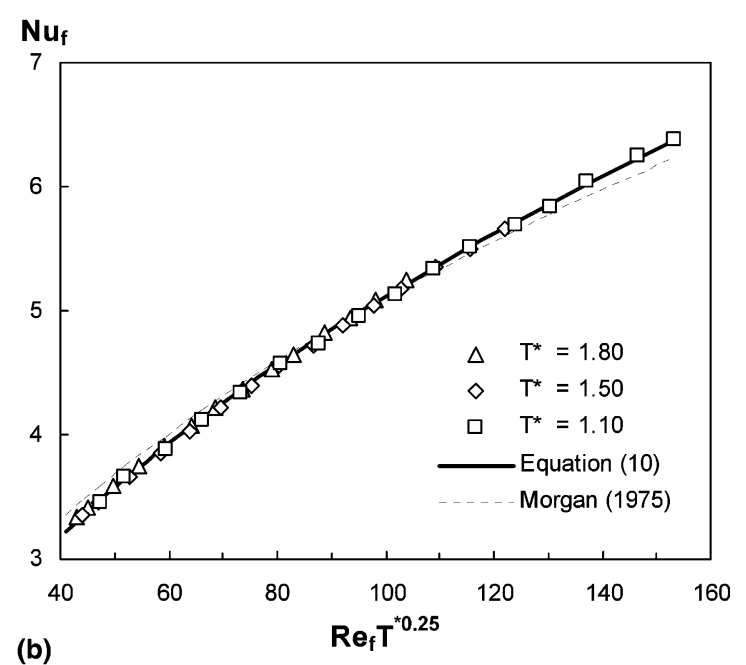

(b)

Fig. 5. $N u_{\mathrm{f}}-R e_{\mathrm{f}} T^{* 0.25}$-relationship of PVS from cylinder model A in (a) steady recirculation regime and (b) periodic vortex regime.

interpretation could be made since the low bound of present data is $R e_{\mathrm{f}}=4.5$ and natural convection is not considered here. The empirical formulas from [3] were also calculated for $T^{*}=1.1$ and 1.8 , respectively. These results are displayed as the dashed lines in Fig. 5(a) and agree quite well with the present data. The maximum deviation of Eq. (9) and Morgan's empirical formula is less than $2.9 \%$.

It is worthy to note here that the form of Eq. (9) is a bit different from that of Eq. (5) that is commonly used in the literature. Basically, the form of Eq. (5) has assumed that (a) there are two variables $\operatorname{Re}_{\mathrm{f}}$ and $F$ (or actually $T^{*}$ ) that are independent of each other, and (b) the Nusselt number should be zero if $T^{*}=0$. However, the Nusselt number has been found to be dependent on the combined variable of $\operatorname{Re}_{\mathrm{f}} T^{* 0.25}$ in the literature. This fact has also been shown in Fig. 5. Moreover, since $T^{*}>1$ is considered here, assumption (b) for Eq. (5) need not be imposed to fit the experimental data. As a result, we have used the relationship of Nusselt number consisting of a power term of one combined variable $R e_{\mathrm{f}} T^{* 0.25}$ and a constant term. In addition, instead of determining the value from the curve fitting, we adopted the power of 0.5 from our experience and also from the theoretical physics for the laminar wake flow.

Similar process has been repeated for processing the data in the periodic wake regime and the results are shown in Fig. 5(b). The fitted heat transfer equation could be expressed as

$N u_{\mathrm{f}}=-0.153+0.527\left(\operatorname{Re}_{\mathrm{f}} T^{* 0.25}\right)^{0.5}$,

for $R e_{\text {eff }} \geqslant R e_{\text {C.eff }}$ (or approximately $R e_{\mathrm{f}} T^{* 0.25} \geqslant 45 \pm 2$ ) and $1.1 \leqslant T^{*} \leqslant 1.8$. In Fig. 5(b), all experimental data collapse excellently on Eq. (10) displayed as the solid line. The maximum and S.D. of experimental data from
Eq. (10) are $0.9 \%$ and $0.4 \%$, respectively. The dashed line in Fig. 5(b) is again the results from [3] for $T^{*}=1.1$ and 1.8. The maximum deviation between Eq. (10) and that of [3] is less than $3.5 \%$.

Since the effective temperature appears to be more physically motivated than the film temperature in [8], the data in Figs. 6(a) and (b) were recalculated based on $T_{\text {eff }}$ instead of $T_{\mathrm{f}}$. Figs. 6(a) and (b) reveal the $N u_{\mathrm{eff}}{ }^{-}$ $R e_{\text {eff }} T^{* 0.25}$-relationship for the steady recirculation flow regime and the periodic wake regime, respectively. Similar to the results in Figs. 5(a) and (b), all experimental data collapse quite well onto the fitted curves for all temperature ratios of $1.1,1.5$ and 1.8. The forced convection for the heated circular cylinder in the steady recirculation regime could be described as

$N u_{\text {eff }}=0.515+0.436\left(R e_{\text {eff }} T^{* 0.25}\right)^{0.5}$

for $R e_{\text {eff }}<R e_{\text {C,eff }}(=47.5 \pm 0.7)$ and $1.1 \leqslant T^{*} \leqslant 1.8$. The maximum and S.D. of experimental data from Eq. (11) are $1.9 \%$ and $1.1 \%$, respectively. The forced convective heat transfer in the periodic wake regime could be described as

$N u_{\text {eff }}=-0.193+0.530\left(R e_{\text {eff }} T^{* 0.25}\right)^{0.5}$,

for $R e_{\text {eff }} \geqslant R e_{\mathrm{C} \text {,eff }}$ and $1.1 \leqslant T^{*} \leqslant 1.8$, and the maximum and S.D. of experimental data from Eq. (12) are $0.9 \%$ and $0.4 \%$, respectively.

Generally speaking, the deviations of experimental data from Eqs. (11) and (12) using the effective temperature are almost the same as those of Eqs. (9) and (10) using conventional film temperature. From the derived universal $S t-R e_{\text {eff }}$-relationship in [8] and the present successful results in Figs. 5(a) and (b), the effective temperature seems a proper characteristic temperature for describing not only fluid dynamics but also heat 

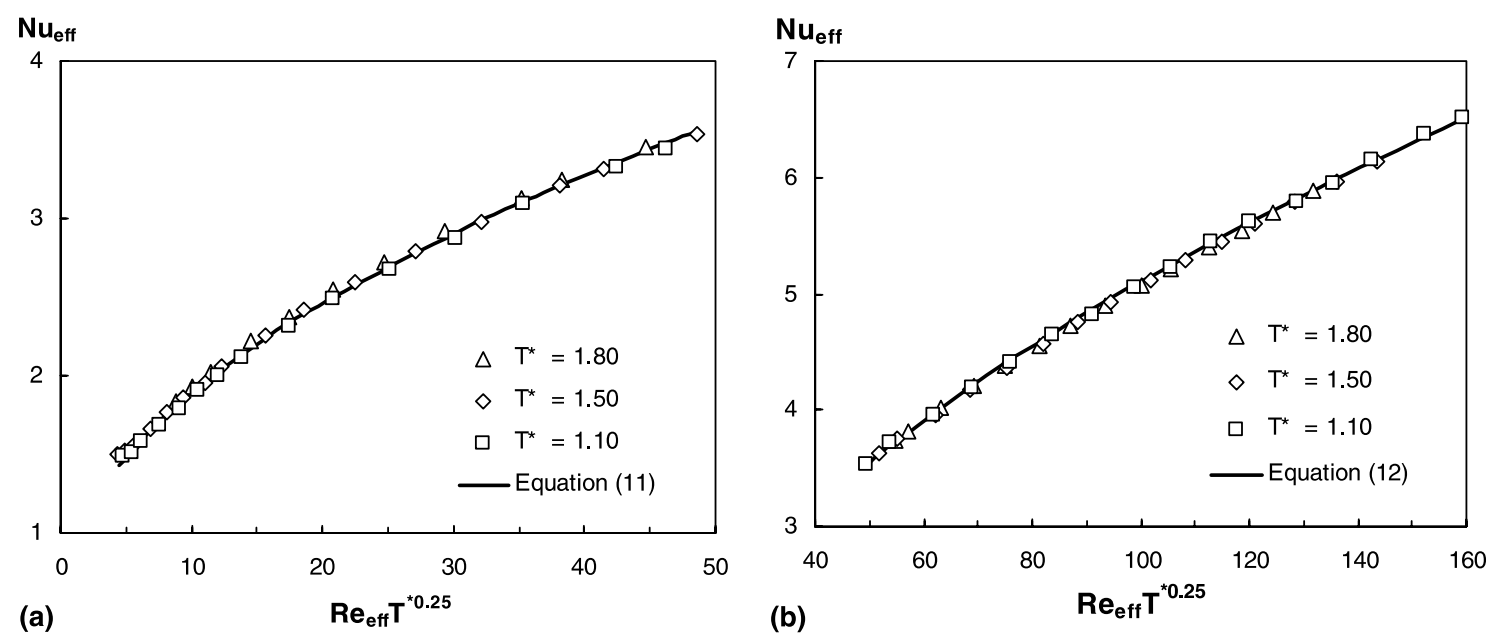

Fig. 6. Relationship of $N u_{\mathrm{eff}}-R e_{\mathrm{eff}} T^{* 0.25}$ for (a) steady recirculation regime and (b) periodic vortex regime.

transfer phenomena of the flow around a heated circular cylinder.

\subsection{Effects of three-dimensional wake on the heat transfer of a heated cylinder}

To study the influence of three-dimensional wake patterns on the heat transfer characters of a heated circular cylinder in crossflow, we start from the generation of the OVS and then focus on the change of Nusselt-Reynolds number-relationship from the PVSto the OVS-modes. Model B was used for the study of OVS under different temperature ratios in the present investigation. The typical smoke-wire visualization of the OVS for heated cylinder is shown in Fig. 7. The surface temperature of the cylinder is $274^{\circ} \mathrm{C}$ and Reynolds number is 96 in this figure. The flow is from right to the left. The corresponding effective Reynolds number and temperature ratio are $R e_{\text {eff }}=67$ and $T^{*}=1.8$.

Following the same representation forms in Section 3.1, Fig. 8 shows the variation of the $N u_{\mathrm{f}}$ against the $R e_{\mathrm{f}} T^{* 0.25}$ for PVS as well as OVS data in both steady recirculation regime and vortex shedding regime under three temperature ratios $T^{*}=1.10,1.50$ and 1.80 , respectively. The Nusselt number was calculated from Eq. (7). The empty symbols represent the experimental data from

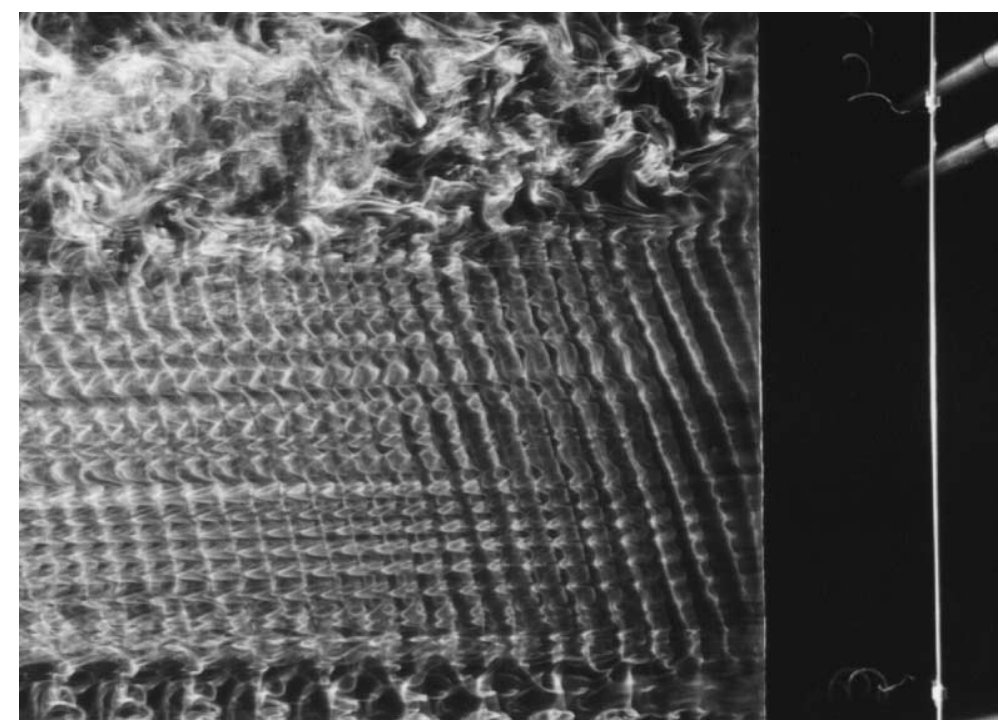

Fig. 7. Smoke-wire visualization of oblique vortex shedding mode from a heated cylinder, $R e_{\mathrm{eff}}=67, T^{*}=1.8\left(R e=96, T_{\mathrm{W}}=274^{\circ} \mathrm{C}\right)$. 


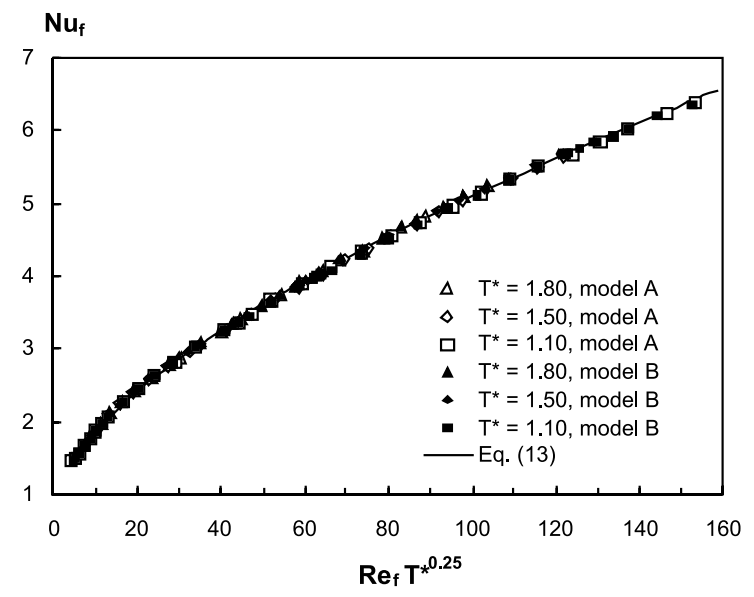

Fig. 8. A second-order correlation for $N u_{\mathrm{f}}-\operatorname{Re}_{\mathrm{f}} T^{* 0.25}$-relationship.

model A and solid symbols from model B. Fig. 8 shows clearly that the heat transfer characters for models A and $B$ are basically the same within the experimental uncertainties. Mean differences of $N u_{\mathrm{f}}$-data from models $\mathrm{A}$ and $\mathrm{B}$ are less than $1 \%$ in the whole studied $R e_{\mathrm{f}}$-range. This means that, in a laminar crossflow, the influence of the three-dimensionality of wake pattern on the heat transfer of a heated circular cylinder could be neglected. This result indicates that a change of vortex shedding frequency and a spanwise flow at the base of a cylinder caused by the change of vortex shedding mode do not influence the mean heat transfer of the heated cylinder in the laminar crossflow. This result confirms the fact that the experimental heat-loss conditions for both models A and B are basically the same. For easy engineering applications, all experimental data from both models A and B are further fitted using a single continuous correlation equation. A threeterm truncated series with the variable of $\left(R e_{\mathrm{f}} T^{* 0.25}\right)^{0.5}$ is used. The best-fit correlation equation is obtained as

$N u_{\mathrm{f}}=0.670+0.349\left(R e_{\mathrm{f}} T^{* 0.25}\right)^{0.5}+0.0094\left(R e_{\mathrm{f}} T^{* 0.25}\right)$,

for $R e_{\mathrm{f}}=4.5-150$ and $T^{*} \leqslant 1.8$. The maximum and S.D. of experimental data from Eq. (13) are $2.1 \%$ and $0.8 \%$, respectively.

Similarly, based on the effective temperature concept, the data in Fig. 8 can be replotted in the form of $N u_{\text {eff }}$ $R e_{\text {eff }} T^{* 0.25}$-relationship as shown in Fig. 9. A continuous $N u_{\text {eff }}-R e_{\text {eff }} T^{* 0.25}$-correlation equation is derived as

$N u_{\text {eff }}=0.721+0.342\left(R e_{\text {eff }} T^{* 0.25}\right)^{0.5}+0.0093 R e_{\text {eff }} T^{* 0.25}$,

for $R e_{\text {eff }}=4.6-155, T^{*} \leqslant 1.8$. The maximum and S.D. of experimental data from Eq. (14) are $3.4 \%$ and $1.1 \%$, respectively.

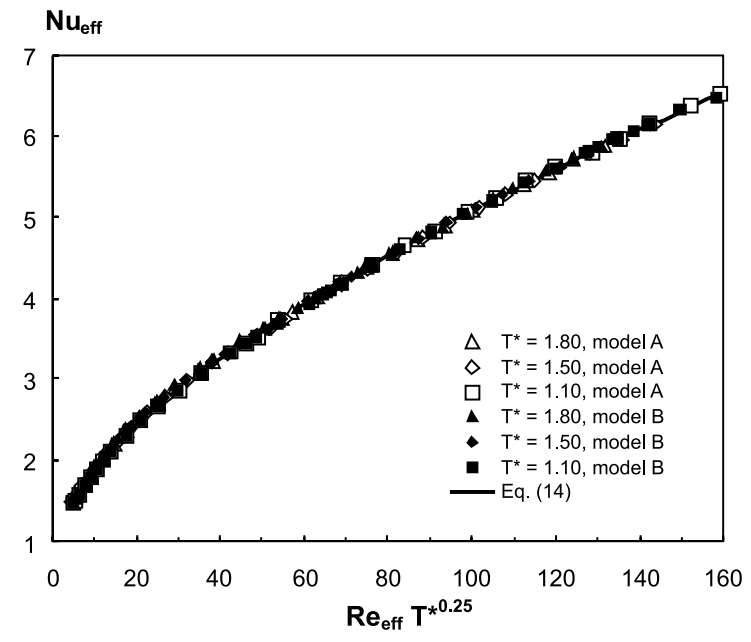

Fig. 9. A second-order correlation for $N u_{\text {eff }}-R e_{\text {eff }} T^{* 0.25}$ relationship.

\subsection{Elimination of the temperature ratio for a linear heat transfer correlation equation of a heated cylinder}

Although all the experimental data could be collapsed excellently on the correlation equations described in the previous sections, from practical viewpoint, a further simplified form without the temperature ratio in the heat transfer correlation equation(s) would be most useful. The reason is that there would be only two variables left in the equation: Nusselt number (the dependent variable) and Reynolds number (the only one independent variable). Different reference temperatures have been proposed in the literature but none seem to have been successfully implemented.

In view of the successful collapse of experimental data on the curves of Eqs. (9) and (10), the abovementioned objective appears likely. To absorb the temperature ratio as an implicit form, a new representative Reynolds number $R e_{\text {rep }}$ is proposed by the following transformation equation, i.e.:

$R e_{\text {rep }}=R e_{\mathrm{f}} T^{* 0.25}$,

where $R e_{\text {rep }}=U_{\infty} d / v_{\text {rep }}$. The $v_{\text {rep }}$ is evaluated by a newly proposed representative temperature $T_{\text {rep }}$ that has a similar form of Eq. (1) as all the other reference temperatures, but with an unknown coefficient of $c_{\text {rep }}$, i.e.

$\frac{T_{\text {rep }}}{T_{\infty}}=1+c_{\text {rep }} \frac{T_{W}-T_{\infty}}{T_{\infty}}$.

By introducing the power proportionality relation $v \sim T^{1.7774}$ from [28], the $c_{\text {rep }}$ could be then derived as a function of $T^{*}$ as

$c_{\text {rep }}=\frac{1+T^{*}-2 T^{* p}}{2 T^{* p}\left(T^{*}-1\right)}$, 


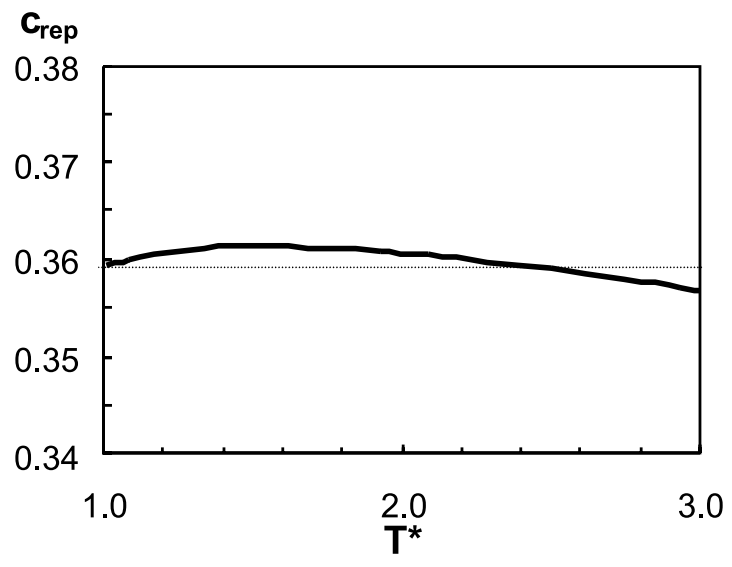

Fig. 10. Relationship of $c_{\text {rep }}$ vs. $T^{*}$.

where the exponent $p$ is a constant of 0.1407 . Fig. 10 shows that the $c_{\text {rep }}$-values are nearly constant for the whole investigated $T^{*}$-range $\left(T^{*} \leqslant 1.8\right)$ and vary only slightly for the wider $T^{*}$-region from 1.0 up to 3.0. If the $c_{\text {rep }}$-value is taken as 0.36 , the deviations of all $c_{\text {rep }}$-values calculated from Eq. (17) and the value of 0.36 are within -0.2 to $0.4 \%$ for the investigated region $\left(T^{*} \leqslant 1.8\right)$.

With Eqs. (15)-(17), the heat transfer correlation of Eqs. (9) and (10) could be transformed as the $N u_{\mathrm{f}}-R e_{\text {rep }}-$ relationship in the following simple form:

$N u_{\mathrm{f}}=0.502+0.434 R e_{\mathrm{rep}}^{0.5}$

for steady recirculation flow regime, and

$N u_{\mathrm{f}}=-0.153+0.527 R e_{\mathrm{rep}}^{0.5}$

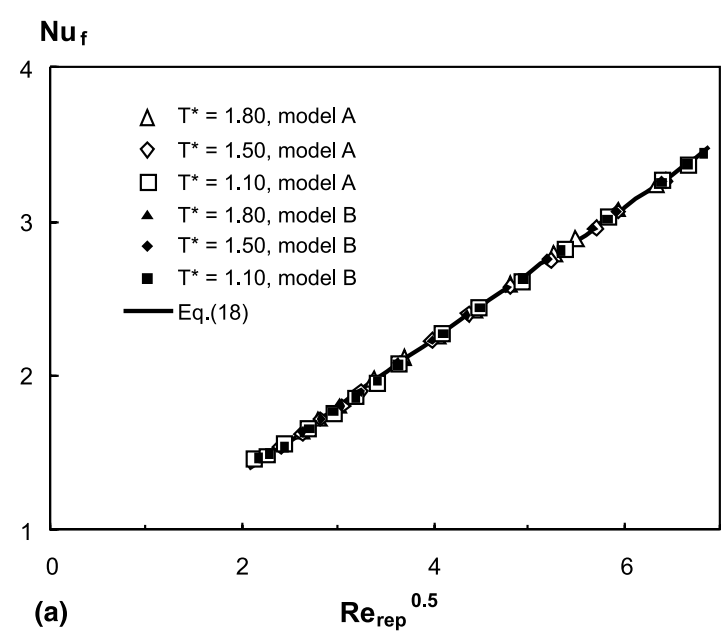

for flows in the periodic wake regime. The validity range as well as the accuracy of Eqs. (18) and (19) are the same as those for Eqs. (9) and (10), respectively. Of course, to simplify the derivation process, the $R e_{\text {rep }}$-value could also be directly calculated based on the approximation equation of the representative temperature:

$\frac{T_{\text {rep }}}{T_{\infty}}=1+0.36 \frac{T_{W}-T_{\infty}}{T_{\infty}}$,

for the kinematic viscosity of the fluid. Thus by using the representative temperature, the heat transfer equations in the laminar region are successfully expressed in a form without the temperature ratio. Moreover, as shown in Eqs. (18) and (19), if the $R e_{\text {rep }}^{0.5}$ is adopted as the independent variable, the Nusselt number becomes linearly proportional to the only variable parameter. Fig. 11(a) shows the excellent collapse of all experimental data (both PVS and OVS) on the linear correlation curve of Eq. (18) in the laminar steady recirculation regime. Similarly, Fig. 11(b) displays the excellent collapse of all experimental data (both PVS and OVS) on another linear correlation curve of Eq. (19) in the regime of the laminar vortex shedding. The slope of Eq. (19) is a bit higher than that of Eq. (18). This is consistent with the fact that the heat transfer coefficient of forced convection increases a bit quicker with the increasing Reynolds number in the periodic regime than that in the steady recirculation regime.

\section{Conclusions}

Forced convective heat transfer from a heated cylinder in laminar crossflow has been experimentally investigated in this paper. Excellent agreement was found between the present experimental data and those in the

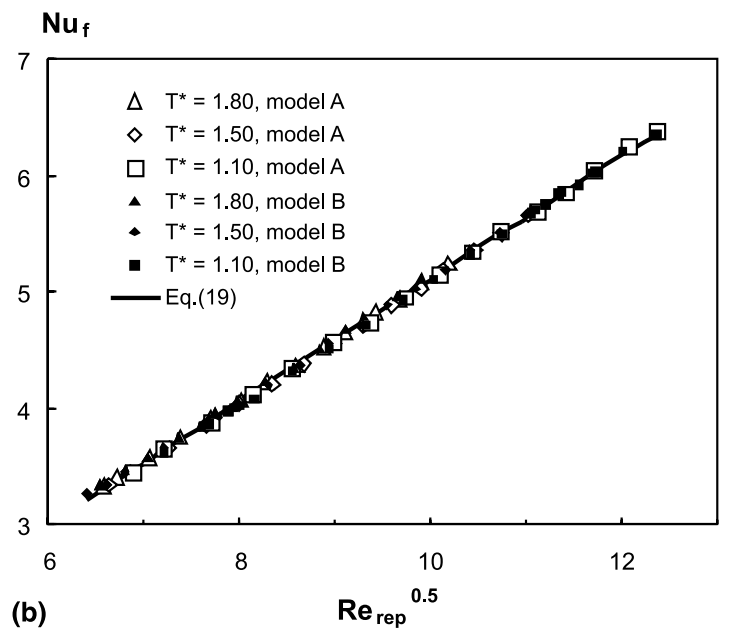

Fig. 11. Linear relationship of $N u_{\mathrm{f}}$ and $R e_{\text {rep }}^{0.5}$ for (a) steady recirculation flow regime and (b) periodic regime. 
literature. The effective temperature concept that was recently introduced for describing the parallel vortex shedding from a heated cylinder has been used to express the heat transfer relationship for the first time. The present result showed that the effective temperature could also be used to describe the heat transfer correlation as well.

The vortex shedding modes, i.e. the parallel vortex shedding and the OVS modes, have significantly changed the shedding frequency of a circular cylinder in the laminar flow regime. However, the influence of different vortex shedding modes on the heat transfer of a heated cylinder was found to be negligible within the experimental uncertainty. All experimental data, not only the parallel but also the OVS, excellently collapsed on the same derived correlation curves in the laminar flow regime for $1.1 \leqslant T^{*} \leqslant 1.8$.

By using a "representative Reynolds number," the heat transfer correlation equation was further expressed in a $T^{*}$-independent form. This newly proposed "representative Reynolds number" was derived on the basis of an approximation equation of "representative temperature $T_{\text {rep }}$ ", which is defined as $T_{\text {rep }} / T_{\infty}=1+$ $0.36\left(T_{\mathrm{W}}-T_{\infty}\right) / T_{\infty}$. A new linear form for the $N u_{\mathrm{f}}-R e_{\text {rep }}^{0.5}$ relationship has been proposed, i.e. $N u_{\mathrm{f}}=0.502+$ $0.434 R e_{\text {rep }}^{0.5}$ for steady recirculation flow regime, and $N u_{\mathrm{f}}=-0.153+0.527 R e_{\text {rep }}^{0.5}$ for flows in the periodic wake regime.

\section{Acknowledgements}

We gratefully acknowledge the support by the National Science Council, Taiwan (NSC 88-2212-E-002053), a partial support by the Ministry of Education, Youth and Gym of the Czech Republic (OC F1.40) under the international Grant of European Cooperation in the Field of Scientific and Technical Research (COST Action F1, CZ.3) and editorial corrections by Prof. K.-C. Wu.

\section{References}

[1] A. Zukauskas, J. Ziugdza, Heat Transfer of a Cylinder in Crossflow, Hemisphere, Washington, DC, 1985.

[2] C.F. Lange, F. Durst, M. Breuer, Momentum and heat transfer from cylinders in laminar crossflow at $10^{-4} \leqslant R e \leqslant 200$, Int. J. Heat Mass Transfer 41 (22) (1998) 3409-3430.

[3] V.T. Morgan, The overall convective heat transfer from smooth circular cylinders, Adv. Heat Transfer 11 (1975) 199-264.

[4] A.P. Hatton, D.D. James, H.W. Swire, Combined forced and natural convection with low-speed air flow over horizontal cylinders, J. Fluid Mech. Part 142 (1970) 17-31.
[5] D.C. Collis, M.J. Williams, Two-dimensional convection from heated wires at low Reynolds numbers, J. Fluid Mech. 6 (1959) 357-384.

[6] W.H. McAdams, Heat Transmission, third ed., McGrawHill, Kogakusha, Tokyo, 1954.

[7] W.J.M. Douglas, S.W. Churchill, Recalculation of data for convective heat transfer between gases and single cylinder with large temperature differences, Chem. Eng. Prog. 52 (18) (1956) 23-28.

[8] A.-B. Wang, Z. Trávníček, K.-C. Chia, On the relationship of Effective Reynolds number and Strouhal number for the laminar vortex shedding of a heated circular cylinder, Phys. Fluids 12 (6) (2000) 1401-1410.

[9] J.C. Lecordier, L. Hamma, P. Paranthoën, The control of vortex shedding behind heated circular cylinders at low Reynolds number, Exp. Fluids 10 (1991) 224-229.

[10] F. Dumouchel, J.C. Lecordier, P. Paranthoën, The effective Reynolds number of a heated cylinder, Int. J. Heat Mass Transfer 41 (12) (1998) 1787-1794.

[11] C.H.K. Williamson, Defining a universal and continuous Strouhal-Reynolds number relationship for the laminar vortex shedding of a circular cylinder, Phys. Fluids 31 (1988) 2742-2745.

[12] C.H.K. Williamson, Vortex dynamics in the cylinder wake, Annu. Rev. Fluid. Mech. 28 (1996) 477-539.

[13] H. Eisenlohr, H. Eckelmann, Vortex splitting and its consequences in the vortex street wake of cylinders at low Reynolds number, Phys. Fluids A 1 (2) (1989) 189-192.

[14] C.H.K. Williamson, Oblique and parallel modes of vortex shedding in the wake of a circular cylinder at low Reynolds numbers, J. Fluid Mech. 206 (1989) 579-627.

[15] D. Hammache, M. Gharib, An experimental study of the parallel and oblique vortex shedding from circular cylinder, J. Fluid Mech. 232 (1991) 567-590.

[16] Y. Yahagi, Structure of two dimensional vortex behind a highly heated cylinder, Trans. JSME B 64 (622) (1998) 209215.

[17] C.H.K. Williamson, G.L. Brown, A series in $(1 / \sqrt{R e})$ to represent the Strouhal-Reynolds number relationship of the cylinder wake, J. Fluids Struct. 12 (1998) 1073-1085.

[18] G.K. Sharma, S.P. Sukhatme, Combined free and forced convection heat transfer from a heated tube to a transverse air stream, Trans. ASME J. Heat Transfer 91 (1969) 457-459.

[19] J.O. Hinze, Turbulence, second ed., McGraw-Hill, New York, 1975.

[20] J. Hilsenrath, Y.S. Touloukian, The viscosity, thermal conductivity, and Prandtl number for air, $\mathrm{O}_{2}, \mathrm{~N}_{2}, \mathrm{NO}, \mathrm{H}_{2}$, $\mathrm{CO}, \mathrm{CO}_{2}, \mathrm{H}_{2} \mathrm{O}, \mathrm{He}$ and A, Trans. ASME 76 (1954) 967-985.

[21] R.M. Fand, K.K. Keswani, A continuous correlation equation for heat transfer from cylinders to air in crossflow for Reynolds numbers from $10^{-2}$ to $2 \times 10^{5}$, Int. J. Heat Mass Transfer 15 (3) (1972) 559-562.

[22] R. Hilpert, Wärmeabgabe von geheizten Drähten und Rohren im Luftstrom, Forsch. Gebiete Ingenieurw. 4 (1933) 215-224.

[23] S.W. Churchill, M. Bernstein, A correlating equation for forced convection from gases and liquids to a circular cylinder in crossflow, Trans. ASME J. Heat Transfer 99 (2) (1977) 300-306.

[24] A.-B. Wang, Y.-Y. Chao, Mean velocity calibration at low airspeed, in: Proceedings of the 2nd Conference of the 
Chinese Metrology Society, Taipei, Taiwan, ROC, 1997, pp. 225-232.

[25] E.R.G. Eckert, R.M. Drake Jr., Analysis of Heat and Mass Transfer, McGraw-Hill, Washington, DC, 1972.

[26] A.E. Perry, in: Hot-Wire Anemometry, Clarendon Press Oxford, Oxford, 1982, p. 10.
[27] F.P. Incropera, D.P. DeWitt, in: Fundamentals of Heat and Mass Transfer, Wiley, New York, 1985, p. 572.

[28] R.J. Goldstein, H.H. Cho, A review of mass transfer measurements using naphthalene sublimation, Exp. Thermal Fluid Sci. 10 (1995) 416-434. 